\title{
STATISTICAL PROPERTIES OF LARGE MESOSPHERIC ELECTRIC FIELDS
}

\author{
S. I. Martynenko \\ Department of Space Radio Physics, Kharkiv V. Karazin National University, \\ 4 Svoboda Square, Kharkiv, 61077 Ukraine, \\ e-mail: Sergey.I.Martynenko@univer.kharkov.ua
}

\begin{abstract}
The statistical analysis of the data on large mesospheric electric field intensities has been undertaken by using the data inferred from MF radar measurements made in the $60-66 \mathrm{~km}$ range over Kharkiv during 1978 - 1994. The analysis has revealed that there exist at least two mechanisms for the generation of large mesospheric electric fields, the most probable of which, with a probability of $70 \%$, can be the summation of random fields from a large number of elementary small-scale mesospheric generators, which results in a one-parameter Rayleigh distribution of large mesospheric electric fields with a mean of approximately $0.7 \mathrm{~V} / \mathrm{m}$. The second mechanism of unknown yet nature results in a sporadic appearance of large mesospheric electric fields, with a probability of $5 \%$ and a mean of approximately $4 \mathrm{~V} / \mathrm{m}$. Statistically significant seasonal variations in the averaged large mesospheric electric fields have not been established. The probability of the absence of the locally generated large mesospheric electric fields amounts to approximately $25 \%$.
\end{abstract}

Key words: large mesospheric electric fields, MF radar/partial reflections, lower ionosphere

\section{Introduction}

The important role of electric fields in middle atmosphere electrodynamics has already been indicated long ago (see, e.g., [Chalmers, 1967]). Initially, the middle atmosphere was considered to be a passive element in the global electric circuit. Bragin et al., [1974], Tyutin [1976], and Kocheev et al., [1976] were the first to declare the detection of large V/m mesospheric electric fields that were detected by in situ rocket measurements. Then the existence of large mesospheric electric fields in the different parts of the world was reported by other workers in the field (see, e.g., review by Goldberg [1984]). The reports mentioned above have generated much discussion on the probability of the appearance of large mesospheric electric fields (Goldberg, 1984; Maynard et al., 1984; Kelley et al., 1983; and Goldberg, 1990). The discovery in recent years of the phenomena red sprites, blue jets, and elves occasionally encountered in the middle atmosphere and caused by high-altitude electric discharges serves an additional experimental confirmation of the existence of large mesospheric electric fields (see, e.g., review by Rodger, [1999]).

Polyakov et al., [1990] have suggested a mechanism for the generation of large mesospheric electric fields directly in the mesosphere. It is based on the two-stream instability driven by the dynamic processes in the medium containing aerosols and charged particles in addition to the ordinary neutral species. This mechanism results in the creation of small-scale, of order of one meter, elementary sources whose fields add up to create small-scale electric fields of hundreds of $\mathrm{V} / \mathrm{m}$ in the mesosphere. The further theoretical studies have shown that the application of electrohydrodynamics to the investigation of inherent in the mesosphere mechanisms for generating electric fields has turned out to be fruitful (see, e.g., Mareev [1998]).

A few experiments have revealed an association between the appearance of large mesospheric electric fields and heavy thunderstorm activity, which could present evidence for the existence of an induction mechanism for the generation of large mesospheric electric fields (see, e.g., Goldberg [1984], Maynard et al., 1984, Goldberg [1990]). In addition, the induction mechanism apparently plays an important role in the development of red sprite phenomenon (see, e.g., Rodger [1999]). Theoretical estimates and experiments show that the characteristic time scale for the relaxation of large mesospheric electric fields in the troposphere-stratosphere-mesosphere subsystem is relatively small and lies within a millisecond-second range, depending on atmospheric-ionospheric conditions, on the mechanism for the 
generation, and on the source location of large mesospheric electric fields (see, e.g., Rodger [1999], Polyakov et al., [1990], Roussel-Dupre and Gurevich [1996]).

The major drawbacks that in situ rocket measurements of large mesospheric electric fields have been their high cost and occasional sampling, which have prevented from collecting a database large enough for reliable statistical analysis to be done. To overcome this problem, a technique for probing large mesospheric electric fields with ground-based MF radars has been advanced and tested at Kharkiv V. Karazin National University (Gokov and Martynenko, [1997], Martynenko [1999], Martynenko et al., [1999]). The experiments have revealed the presence of large mesospheric electric fields in $70 \%$ of the cases when the partially reflected signals were observed from the $60-66 \mathrm{~km}$ altitude range. The electric current density averaged over all the sample observations does not practically depend on altitude that permits the conclusion to be made that the source of large mesospheric electric fields can be represented by the current source in the given altitude region (Martynenko, Rozumenko, and Tyrnov [2001]). As a result, new capabilities have appeared for specifying the existing models for large mesospheric electric fields. The aim of this paper is an estimate of basic parameters of large mesospheric electric fields by doing statistical analysis of the data that have formerly been used by Gokov and Martynenko, [1997], Martynenko, [1999], Martynenko et al., [1999], Martynenko, Rozumenko, and Tyrnov [2001].

\section{Distribution of Large Mesospheric Electric Field Intensities}

As the input data for the statistical analysis, we use the database on large mesospheric electric fields augmented by MF radar effective electron collision frequency, $v_{e}$, data acquired from the $z=60-$ $66 \mathrm{~km}$ range at Kharkiv V. Karazin National University during 1978 - 1994 by Gokov and Martynenko [1997] and Martynenko et al. [1999] The MF radar operated at 1.8 - 3.0-MHz with a 25-micros pulse length and a 1 per second repetition rate. The primary $v_{e}$ sample size is $n=185$. Preliminary data analysis included the estimate of the histogram showing the distribution of $v_{e} / v_{e m}$ where $v_{e m}$ is a model value of $v_{e}$ in the absence of large mesospheric electric fields at heights from which the partially reflected signals were received. Allowing for the kinetic effects, Gokov and Martynenko [1997] and Martynenko et al. [1999] assumed $v_{e m}=3.75 \times 10^{7} \mathrm{~s}^{-1}$ at $z=60 \mathrm{~km}, v_{e m}=2.55 \times 10^{7} \mathrm{~s}^{-1}$ at $z=63 \mathrm{~km}$, and $v_{e m}=1.68 \times 10^{7} \mathrm{~s}^{-1}$ at $z=66 \mathrm{~km}$. It is obvious that the $v_{e} / v_{e m}$ sample should have a Gaussian distribution with a mean of $M\left[v_{e}\right.$ $\left./ v_{e m}\right]=1$. This provides the reason for suggesting that the values $v_{e} / v_{e m}<1$ are associated with cases when large mesospheric electric fields are absent, and these data should be excluded from the database on $v_{e} / v_{e m}$ that is intended for revealing large mesospheric electric field effects. Furthermore, the symmetry of the Gaussian distribution of $v_{e} / v_{e m}$ about $v_{e} / v_{e m}=1$ in the absence of large mesospheric electric fields provides another criterion for excluding the undisturbed component with $v_{e} / v_{e m}>1$ from the analysis. The database on $v_{e} / v_{e m}$ observed in the presence of large mesospheric electric fields, which was collected in the described above fashion, was processed with the technique of Gokov and Martynenko [1997], Martynenko [1999], and Martynenko et al. [1999] in order to obtain the histogram for the distribution, $w_{E}$, of the large mesospheric electric fields, $E$, and the result is shown in Figure 1. Here, $w_{E}=n_{i} /(n \Delta E), i$ is the number of a current cell, $i=1, \ldots, 13, n_{i}$ is the number of realizations of $E$ that lie within the $(i-1) \Delta E$ $<\mathrm{E} \leq i \Delta E$ cell, $\Delta E=0.5 \mathrm{~V} / \mathrm{m}$ is the width of each cell, and $n=139$ is the sample size. Within the 0.99 confidence interval, the histogram exhibits the possibility of dividing it into two parts: that constituting the main body $\mathrm{E} \leq 2.5 \mathrm{~V} / \mathrm{m}, n=129$, and that constituting the tail $E>2.5 \mathrm{~V} / \mathrm{m}, n=10$. Also, within the 0.99 confidence interval estimated by making use of Pearson's test, the main body corresponds to oneparameter Rayleigh probability density functions as given by

$$
f(E)=\frac{E}{\sigma^{2}} e^{-\frac{E^{2}}{2 \sigma^{2}}}
$$




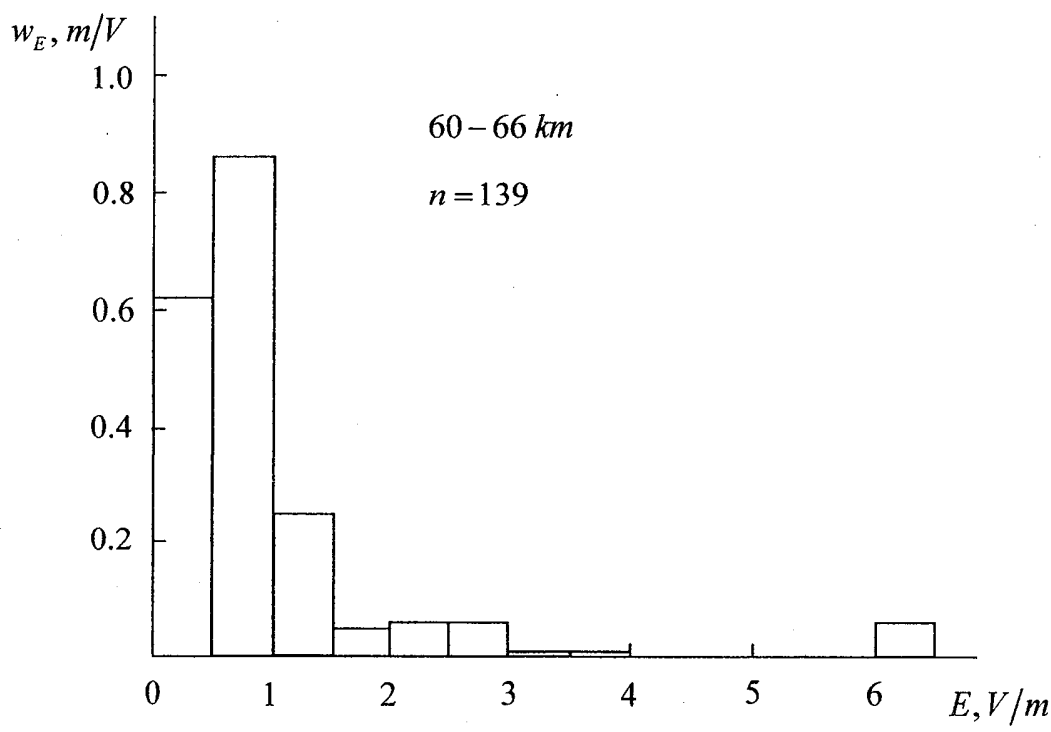

Fig. 1. Histogram showing the distribution, $w_{E}$, of the large mesospheric electric fields, $E$.

where $\sigma=E_{m}$ is the most probable value of $E, M_{1}[E]=(\pi / 2)^{1 / 2} \sigma$ is the mean, $M_{2}[E]=2 \sigma^{2}$ is the second ordinary moment for the Rayleigh set, and $D[E]=(2-\pi / 2) \sigma^{2}$ is the variance. Within the same 0.99 confidence interval, the Rayleigh part of the histogram provides an estimate of $M_{1}[E]=0.72 \pm 0.11 \mathrm{~V} / \mathrm{m}$ where the sample mean $\left\langle E_{R}>=0.72 \mathrm{~V} / \mathrm{m}\right.$, which corresponds to $\sigma=0.57 \mathrm{~V} / \mathrm{m}$. The corresponding histogram $w_{E}$ for large mesospheric electric field intensities constructed by using $n=129$ samples and the theoretical Rayleigh probability density function are shown in Figure 2.

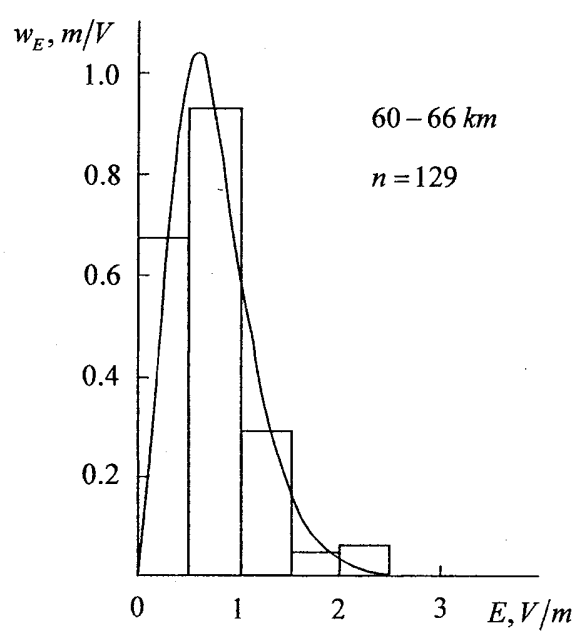

Fig. 2. Histogram, $w_{E}$, showing the Rayleigh part of the distribution of large mesospheric electric field intensities, $E$, and the corresponding theoretical Rayleigh probability density function (continuous curve).

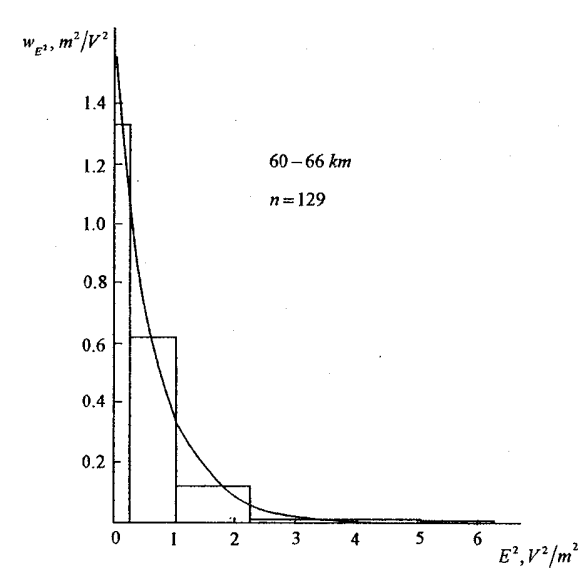

Fig. 3. Histogram, $w_{E^{2}}$, showing the distribution of the squared large mesospheric electric field intensities, $E^{2}$, and the corresponding theoretical exponential distribution shown as continuous curve. 
Hence, there should exist at least two mechanisms for generating large mesospheric electric fields, and the Rayleigh distribution of $E$ can be formed as a result of the summation of the random fields from a large number of prime small-scale mesospheric generators; the possible mesospheric processes resulting in such small-scale active elements have been discussed, e.g., by Goldberg [1990] and Polyakov, Rapoport, and Trakhtengerts [1990]. The treated data have shown that the probability of existence of such an integral Rayleigh mesospheric generator is equal to approximately $70 \%$, and the probability of the lack of large mesospheric electric field is about $25 \%$.

Unfortunately, the number of observations occurring in the sample interval $E>2.5 \mathrm{~V} / \mathrm{m}$ is equal to $n=10$, which is too little for drawing statistical inferences from, and these data are hardly enough only for crudely characterizing a mean of $\langle E\rangle=(4.3 \pm 1.3)$ within the 0.90 confidence interval. As a whole the possible upper bound for $E$ is limited by a threshold value of $E_{t}=218 \times\left(p / p_{0}\right) \mathrm{kV} / \mathrm{m}$, e.g., RousselDupre and Gurevich [1996], when an atmospheric breakdown occur and is associated with red sprite, blue jet, and elf phenomena in the middle atmosphere (here $p$ is the atmospheric pressure at the altitude $z$, $p_{0}$ is the atmospheric pressure at sea level). For example, $E_{t}=50-10 \mathrm{~V} / \mathrm{m}$ within the altitude range of $z=$ $60-70 \mathrm{~km}$, respectively. Using the well-known mathematical technique for obtaining the differential distribution function of a deterministic function of random variable, it is easy, making use of (1), to make a transition from $f(E)$ to the distribution function $f\left(E^{2}\right)$

$$
f\left(E^{2}\right)=\frac{1}{\sigma^{2}} e^{-\frac{E^{2}}{2 \sigma^{2}}},
$$

which is a one-parameter exponential with the parameter $\mu=1 / 2 \sigma^{2}$. Figure 3 depicts the histogram showing the distribution $w_{E^{2}}$, the theoretical Rayleigh probability density function $w_{E}$, and the 0.99 confidence intervals drawn from applying Pearson's test. Here, the range of observations of $E^{2}$ is divided into the following cells of unequal width: $\Delta E_{1}^{2}=0-0.25(\mathrm{~V} / \mathrm{m})^{2}, \Delta E_{2}^{2}=0.25-1(\mathrm{~V} / \mathrm{m})^{2}, \Delta E_{3}^{2}=1-2.25$ $(\mathrm{V} / \mathrm{m})^{2}, \Delta E_{4}^{2}=2.25-4(\mathrm{~V} / \mathrm{m})^{2}, \Delta E_{5}^{2}=4-6.25(\mathrm{~V} / \mathrm{m})^{2}$, which correspond to the cells into which the range of observations of $E$ was divided in Figure 2.

An attempt has been made to ascertain seasonal dependences in the statistical parameters of the large mesospheric electric fields. To arrive at this objective, all the data in the database were arbitrary divided into two subsets: winter (September 24 - March 23) and summer (March 24 - September 23). Then the Rayleigh components in the $E$ distribution were constructed, with $n=69$ for the winter and $n=$ 60 for the summer. The corresponding histograms showing the distributions $w_{E}$ and the theoretical Rayleigh distributions are presented in Figure 4 and Figure 5. Within the 0.99 confidence interval, in accordance with the Pearson's test, the histograms correspond to Rayleigh probability density functions with $\left\langle E_{R}>=0.70 \mathrm{~V} / \mathrm{m}\right.$ and $\sigma=0.56 \mathrm{~V} / \mathrm{m}$ for the winter, and $\left\langle E_{R}>=0.75 \mathrm{~V} / \mathrm{m}\right.$ and $\sigma=0.60 \mathrm{~V} / \mathrm{m}$ for the summer. It can be seen that that the seasonal differences have turned out to be statistically insignificant. The $E>2.5 \mathrm{~V} / \mathrm{m}$ values were also occasionally observed during both the winter $(n=6)$ and the summer $(n$ $=4$ ). This may indicate that, e.g., the mean local seasonal variations in mesospheric parameters, if they exist, and the mean local thunderstorm activity do not exert a noticeable effect at least on the mean performance of the Rayleigh generator of large mesospheric electric fields.

\section{Discussion and Conclusions}

Thus, the statistical analysis of the large mesospheric electric field intensity data acquired with the Kharkiv V. Karazin National University MF radar in the $60-66 \mathrm{~km}$ region over $1978-1994$ interval [Gokov and Martynenko, 1997; Martynenko et al., 1999] resulted in the following inferences.

There are at least two mechanisms for the generation of large mesospheric electric fields in the mesosphere. The most likely of them, with the $70 \%$ probability, is the summation of random fields 


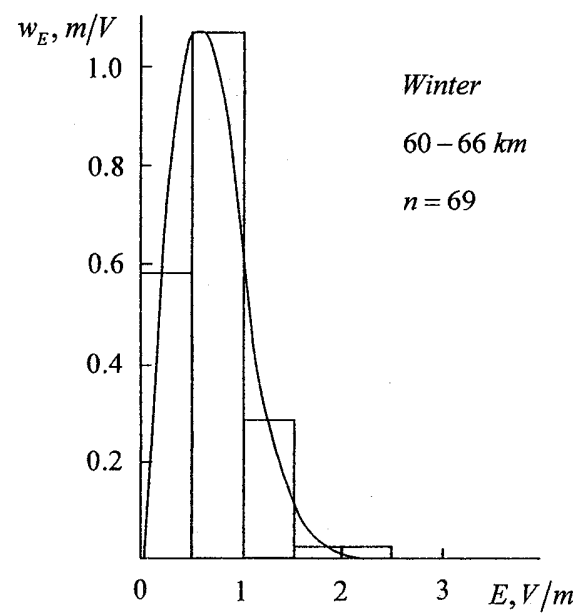

Fig. 4. Histogram, $w_{E}$, showing the Rayleigh part of the distribution of large mesospheric electric field intensities, $E$, for the winter interval, and the corresponding theoretical Rayleigh distribution shown as continuous curve.

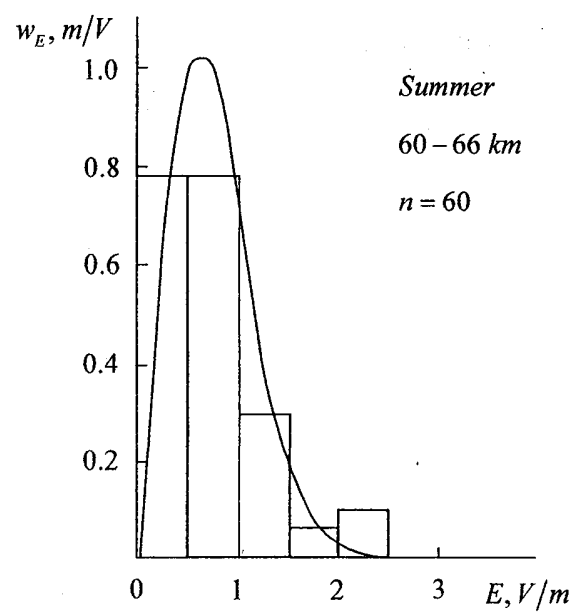

Fig. 5. Histogram, $w_{E}$, showing the Rayleigh part of the distribution of large mesospheric electric field intensities, $E$, for the summer interval, and the corresponding theoretical Rayleigh distribution shown as continuous curve.

from a large number of elementary small-scale mesospheric generators, which results in a one-parameter Rayleigh distribution of the total large mesospheric electric field intensity $E$ with a mean value of approximately $0.7 \mathrm{~V} / \mathrm{m}$ in the $60-66 \mathrm{~km}$ altitude region, or in the corresponding one-parameter exponential distribution of the large mesospheric electric field squared intensity $E^{2}$. The second mechanism of unknown yet nature gives rise to a sporadic, with $5 \%$ probability, appearance of large mesospheric electric field intensities $E>2.5 \mathrm{~V} / \mathrm{m}$ with a mean of $4 \mathrm{~V} / \mathrm{m}$. The statistically significant seasonal differences in the averaged large mesospheric electric field parameters have not been revealed. The probability of the lack of local large mesospheric electric fields amounts to approximately $25 \%$.

It is important to note that in a given experiment it is difficult to establish the cause of the observed small, up to $\pm 20 \%$, variations in the relative electron collision frequency $v_{e} / v_{e m}$ : whether it is random errors, the existence of relatively weak electric fields with the intensities of $E<0.3-0.1 \mathrm{~V} / \mathrm{m}$, the variations of different origins in atmospheric parameters, or some error in the reference undisturbed medium model. Therefore, the probabilities of disturbed and undisturbed conditions in the mesosphere due to large mesospheric electric fields need to be specified.

An essential restriction in applying the technique for remote large mesospheric electric field diagnostics, the results from which are discussed in this paper, is a frequent absence of the signals partially reflected from the $60-70 \mathrm{~km}$ altitude range, particularly under nighttime conditions; this makes it impossible for this technique to continuously monitor large mesospheric electric fields. Therefore, it is further necessary both to improve the existing and to develop new remote diagnostic techniques for monitoring processes mentioned above. As a whole, the results obtained so far can be used for developing a statistical model of disturbances from large mesospheric electric fields in the lower ionosphere.

Acknowledgments. The author is supported by Science and Technology Center in Ukraine Grant 1773 to Kharkiv V. Karazin National University.

\section{References}


Bragin, Yu. A., A. A. Tyutin, A. A. Kocheev, A. A. Tyutin, Direct measurement of the atmospheric vertical electric field intensity up to $80 \mathrm{~km}$, Cosmic Res., vol. 12, no. 2, pp. $306-308,1974$ (in Russian).

Chalmers, J. A., Atmospheric Electricity, 2nd ed., Pergamon Press, New York, 1967.

Gokov, A. M., and S. I. Martynenko, Changes in the electron collision frequency and electric field in the lower ionosphere, Geomagnetism and Aeronomy, vol. 37, no. 2, pp. 76 - 80, 1997 (in Russian).

Goldberg, R. A., Middle atmospheric electrodynamics: status and future, J. Atmos. Terr. Phys., vol. 46, no. 11 , pp. $1083-1101,1984$.

Goldberg, R. A., Middle atmospheric electrodynamics during MAP, Adv. Space Res., vol.10, no. 10, pp. $209-217,1990$.

Kelley, M. C., C. L. Siefring, and R. F. Pfaff, Large amplitude middle atmospheric electric fields: fact or fiction? Geophys. Res. Lett., vol. 10, no.8, pp. 733-736, 1983.

Kocheyev, A. A., L. N. Smirnykh, A. A. Tyutin, Direct simultaneous measurements of the ion number density, electrical conductivity, and atmospheric electric field strength vertical component in the $0-$ $85 \mathrm{~km}$ altitude range, Cosmic Res., vol. 14, no. 1, pp. 148 - 151 (in Russian).

Mareev, E. A., Modern progress in the global electric circuit research, V-th International Suzdal URSI Symp. Modification of Ionosphere (ISSMI'98). Book of Abstracts. IZMIRAN, Moscow, pp. 96 - 97, 1998.

Martynenko, S. I., Atmospheric electric field and disturbances of the lower ionosphere parameters, $J$. Atmos. Electricity, vol. 19, no. 1, pp. 1-9, 1999.

Martynenko S. I., V. T. Rozumenko, A. M. Tsymbal, O. F. Tyrnov, and A. M. Gokov, Mesospheric electric field measurements with a partial reflection radar, J. Atmos. Electricity, vol. 19, no. 2, pp. 81 86, 1999.

Martynenko, S. I., V. T. Rozumenko, and O. F. Tyrnov, New possibilities for mesospheric electricity diagnostics, Adv. Space Res., vol. 27, no. 6-7, pp.1127-1132, 2001.

Maynard, N. C., L. C. Hale, J. D. Mitchell, F. J. Schmidlin, R. A. Goldberg, J. R. Barcus, F. Soraas, and C. L. Groskey, Electrical structure in the high-latitude middle atmosphere, J. Atmos. Terr. Phys., vol. 46, nө. $=9$, pp. $807-817,1984$.

Polyakov, S. V., V. O. Rapoport, and V.Yu. Trakhtengerts, On the generation of electric fields in the upper atmosphere, Geomagnetism and Aeronomy, vol. 30, no. 5, pp. 869 - 871, 1990 (in Russian).

Rodger, C. J., Red sprites, upward lightning, and VLF perturbations, Rev. Geophys., vol. 37, no. 3, pp. 317-336, 1999.

Roussel-Dupre, R., and A. V. Gurevich, On runaway breakdown and upward propagating discharges, $J$. Geophys. Res., vol. 101, no A2, pp. 2297 - 2311, 1996.

Tyutin, A. A., Mesospheric maximum of the electric field strength, Cosmic Res., vol. 14, no. 1, pp. 143 144, 1976 (in Russian).

(Received June 25, 2002; revised July 24, 2002;

accepted August 20, 2002) 